\title{
Autosomal dominant spondylocostal dysostosis
}

INSERM

\section{Source}

INSERM. (1999). Orphanet: an online rare disease and orphan drug data base. Autosomal dominant spondylocostal dysostosis. ORPHA:1797

Autosomal dominant spondylocostal dysostosis is a very rare and mild form of spondylocostal dysostosis characterized by vertebral and costal segmentation defects, often with a reduction in the number of ribs. 